\title{
A Unique Common Fixed Point Theorem for Four Mappings Satisfying Presic Type Condition in Fuzzy Metric Spaces
}

\author{
K.P.R.Rao ${ }^{*}$, A.Som Babu ${ }^{2}$ and Md.Mustaq Ali ${ }^{3}$ \\ ${ }^{1}$ Department of Mathematics, Acharya Nagarjuna University, Nagarjuna Nagar -522 510, A.P., India. \\ ${ }^{2}$ Dept.of Basic Science Humanities, NRI Institute of Technology, Agiripalli,Andhra Pradesh,India. \\ Dept.of Science Humanities, Usharama Engg.College, Telaprolu, Andhra Pradesh,India. \\ Email: kprrao2004@yahoo.com, somu.mphil@gmail.com, alimustaq9@gmail.com
}

\begin{abstract}
In this paper, we obtain a Presic type fixed point theorem for two pairs of jointly $2 k$-weakly compatible maps in fuzzy metric spaces. We also give an example to illustrate our main theorem. We obtain two corollaries for three maps and two maps.
\end{abstract}

Keywords: Fuzzy metric spaces, presic type theorem, jointly $2 k$-weakly compatible mappings.

\section{Introduction and Preliminaries}

There are a number of generalizations of Banach contraction principle. One such generalization is given by S.B.Presic [9] in 1965.

Let $f: X^{k} \rightarrow X$, where $k \geq 1$ is a positive integer. A point $x^{*} \in X$ is called a fixed point of $f$ if $x^{*}=f\left(x^{*}, x^{*}, \cdots, x^{*}\right)$. Consider the $k$-order non linear difference equation

$$
x_{n+1}=f\left(x_{n-k+1}, x_{n-k+2}, \cdots, x_{n}\right) \text { for } n=k-1, k, k+1,
$$

with the initial values $x_{0}, x_{1}, x_{2}, \cdots, x_{k-1} \in X$.

Equation (1.1) can be studied by means of fixed point theory in view of the fact that $x \in X$ is a solution of (1.1) if and only if $x$ is a fixed point of $f$. One of the most important results in this direction is obtained by Presic [9] in the following way. Throughout this paper,let $N$ denote the set of all positive integers.

Theorem 1.1. ([g]) Let $(X, d)$ be a complete metric space, $k$ a positive integer and $f: X^{k} \rightarrow X$. Suppose that

$$
d\left(f\left(x_{1}, x_{2}, \cdots, x_{k}\right), f\left(x_{2}, x_{3}, \cdots, x_{k+1}\right)\right) \leq \sum_{i=1}^{k} q_{i} d\left(x_{i}, x_{i+1}\right)
$$

holds for all $x_{1}, x_{2}, \cdots, x_{k}, x_{k+1} \in X$, where $q_{i} \geq 0$ and $\sum_{i=1}^{k} q_{i} \in[0,1)$. Then $f$ has a unique fixed point $x^{*}$. Moreover, for any arbitrary points $x_{1}, x_{2}, \cdots, x_{k+1}$ in $X$, the sequence $\left\{x_{n}\right\}$ defined by $x_{n+k}=$ $f\left(x_{n}, x_{n+1}, \cdots, x_{n+k-1}\right)$, for all $n \in N$ converges to $x^{*}$.

Later Ciric and Presic [6] generalized the above theorem as follows.

Theorem 1.2. ([6]). Let $(X, d)$ be a complete metric space, $k$ a positive integer and $f: X^{k} \rightarrow X$. Suppose that

$$
d\left(f\left(x_{1}, x_{2}, \cdots, x_{k}\right), f\left(x_{2}, x_{3}, \cdots, x_{k+1}\right)\right) \leq \lambda \max \left\{d\left(x_{i}, x_{i+1}\right): 1 \leq i \leq k\right\}
$$

holds for all $x_{1}, x_{2}, \cdots, x_{k}, x_{k+1}$ in $X$, where $\lambda \in[0,1)$. Then $f$ has a fixed point $x^{*} \in X$. Moreover, for any arbitrary points $x_{1}, x_{2}, \cdots, x_{k+1}$ in $X$, the sequence $\left\{x_{n}\right\}$ defined by $x_{n+k}=f\left(x_{n}, x_{n+1}, \cdots, x_{n+k-1}\right)$, for all $n \in N$ converges to $x^{*}$. Moreover, if $d(f(u, u, \cdots, u), f(v, v, \cdots, v))<d(u, v)$ holds for all $u, v \in X$ with $u \neq v$, then $x^{*}$ is the unique fixed point of $f$.

Recently Rao et al.[4,5] obtained some Presic type theorems for two and three maps in metric spaces. Now we give the following definition of $[4,5]$. 
Definition 1.3. Let $X$ be a non empty set and $T: X^{2 k} \rightarrow X$ and $f: X \rightarrow X$.The pair $(f, T)$ is said to be $2 k$-weakly compatible if $f(T(x, x, \ldots, x, x))=T(f x, f x, \ldots, f x, f x)$ whenever $x \in X$ such that $f x=T(x, x, \ldots, x, x)$.

Using this definition, Rao et al. [4] proved the following

Theorem 1.4. ([4]). Let $(X, d)$ be a metric space, $k$ a positive integer and $S, T: X^{2 k} \rightarrow X, f: X \rightarrow X$ be mappings satisfying:

(1.4.1) $d\left(S\left(x_{1}, x_{2}, \cdots, x_{2 k}\right), T\left(x_{2}, x_{3}, \cdots, x_{2 k+1}\right)\right) \leq \lambda \max _{1 \leq i \leq 2 k}\left\{d\left(f x_{i}, f x_{i+1}\right)\right\}$ for all $x_{1}, x_{2}, \cdots, x_{2 k}, x_{2 k+1}$ in $X$,

$(1.4 .2) d\left(T\left(y_{1}, y_{2}, \cdots, y_{2 k}\right), S\left(y_{2}, y_{3}, \cdots, y_{2 k+1}\right)\right) \leq \lambda \max _{1 \leq i \leq 2 k}\left\{d\left(f y_{i}, f y_{i+1}\right)\right\}$ for all $y_{1}, y_{2}, \cdots, y_{2 k}, y_{2 k+1}$

in $X$, where $0<\lambda<1$

(1.4.3) $d(S(u, \cdots, u), T(v, \cdots, v))<d(f u, f v)$, for all $u, v \in X$ with $u \neq v$

(1.4.4) Suppose that $f(X)$ is complete and either $(f, S)$ or $(f, T)$ is a $2 k$-weakly compatible pair.

Then there exists a unique point $p \in X$ such that $f p=p=S(p, \cdots, p)=T(p, \cdots, p)$.

Recently Murthy and Rashmi [8] defined the following function

Definition 1.5. Let $\phi:[0,1] \longrightarrow[0,1]$ be such that:

(i) $\phi$ is increasing and continuous function in each variable,

(ii) $\phi(t, t, t, \ldots, t) \geq t$ for all $t \in[0,1]$.

Using this function,Murthy and Rashmi [8] extended Theorem 1.4 to fuzzy metric spaces as follows.

Theorem 1.6. ([8]) Let $(X, M, *)$ be a fuzzy metric space and $S, T: X^{2 k} \rightarrow X, f: X \rightarrow X$ be mappings satisfying for each positive integer $k, 0<q<\frac{1}{2}$ and $t \in[0, \infty)$ :

(1.6.1) $M\left(S\left(x_{1}, x_{2}, \ldots, x_{2 k-1}, x_{2 k}\right), T\left(x_{2}, x_{3}, \ldots, x_{2 k}, x_{2 k+1}\right), q t\right) \geq \phi\left(M\left(f x_{1}, f x_{2}, t\right), \cdots, M\left(f x_{2 k}, f x_{2 k+1}, t\right)\right)$ for all $x_{1}, x_{2}, \ldots, x_{2 k+1} \in X$,

(1.6.2) $M\left(T\left(y_{1}, y_{2}, \ldots, y_{2 k-1}, y_{2 k}\right), S\left(y_{2}, y_{3}, \ldots, y_{2 k}, y_{2 k+1}\right), q t\right) \geq \phi\left(M\left(f y_{1}, f y_{2}, t\right), \cdots, M\left(f y_{2 k}, f y_{2 k+1}, t\right)\right)$ for all $y_{1}, y_{2}, \ldots, y_{2 k+1} \in X$,

(1.6.3) $M(S(u, u, \ldots, u, u), T(v, v, \ldots, v, v), q t)>M(f u, f v, t)$

for all $u, v \in X$ with $u \neq v$.

Suppose that $f(X)$ is complete and either $(f, S)$ or $(f, T)$ is $2 k$-weakly compatible pair. Then there exists a unique $p \in X$ such that $p=f p=S(p, p, \ldots, p, p)=T(p, p, \ldots, p, p)$.

In this paper, we obtain a Presic type theorem for four mappings satisfying a slight different contractive condition in fuzzy metric spaces. We also give an example and two corollaries to our main theorem.

First we recall some basic definitions and lemmas which play crucial roles in the theory of fuzzy metric spaces.

Definition 1.7. ([2]). A binary operation $*:[0,1] \times[0,1] \longrightarrow[0,1]$ is a continuous $t$-norm if it satisfies the following conditions:

1. * is associative and commutative,

2. * is continuous,

3. $a * 1=a$ for all $a \in[0,1]$,

4. $a * b \leq c * d$ whenever $a \leq c$ and $b \leq d$, for each $a, b, c, d \in[0,1]$.

Two typical examples of a continuous $t$-norm are $a * b=a b$ and $a * b=\min \{a, b\}$.

Definition 1.8. ([1]). A 3-tuple $(X, M, *)$ is called a fuzzy metric space if $X$ is an arbitrary (non-empty) set, $*$ is a continuous $t$-norm and $M$ is a fuzzy set on $X^{2} \times(0, \infty)$, satisfying the following conditions for each $x, y, z \in X$ and $t, s>0$,

$\left(M_{1}\right) M(x, y, t)>0$, 
$\left(M_{2}\right) M(x, y, t)=1$ if and only if $x=y$,

$\left(M_{3}\right) M(x, y, t)=M(y, x, t)$,

$\left(M_{4}\right) M(x, y, t) * M(y, z, s) \leq M(x, z, t+s)$,

$\left(M_{5}\right) M(x, y,):.(0, \infty) \longrightarrow[0,1]$ is continuous.

Let $(X, M, *)$ be a fuzzy metric space. For $t>0$, the open ball $B(x, r, t)$ with center $x \in X$ and radius $0<r<1$ is defined by $B(x, r, t)=\{y \in X: M(x, y, t)>1-r\}$.

If $(X, M, *)$ is a fuzzy metric space, let $\tau$ be the set of all $A \subset X$ with $x \in A$ if and only if there exist $t>0$ and $0<r<1$ such that $B(x, r, t) \subset A$. Then $\tau$ is a topology on $X$ (induced by the fuzzy metric $M)$. This topology is Hausdorff and first countable.

A sequence $\left\{x_{n}\right\}$ in $X$ converges to $x$ if and only if $M\left(x_{n}, x, t\right) \rightarrow 1$ as $n \rightarrow \infty$, for each $t>0$. It is called a Cauchy sequence in the sense of [7] if $\lim _{n \rightarrow \infty} M\left(x_{n}, x_{n+p}, t\right)=1$, for all $t>0$ and each positive integer $p$. The fuzzy metric space $(X, M, *)$ is said to be complete if every Cauchy sequence is convergent.

Example 1.9. Let $X=[0,1]$ and $a * b=a b$ for all $a, b \in[0,1]$ and let $M$ be the fuzzy set on $X \times X \times(0, \infty)$ defined by $M(x, y, t)=e^{-\frac{|x-y|}{t}}$ for all $t>0$. Then $(X, M, *)$ is a fuzzy metric space.

Lemma 1.10. ([r] $)$. Let $(X, M, *)$ be a fuzzy metric space. Then $M(x, y, t)$ is non-decreasing with respect to $t$, for all $x, y \in X$.

Definition 1.11. ([3]). Let $(X, M, *)$ be a fuzzy metric space.Then $M$ is said to be continuous on $X^{2} \times(0, \infty)$ if $\lim _{n \rightarrow \infty} M\left(x_{n}, y_{n}, t_{n}\right)=M(x, y, t)$, whenever a sequence $\left\{\left(x_{n}, y_{n}, t_{n}\right)\right\}$ in $X^{2} \times(0, \infty)$ converges to a point $(x, y, t) \in X^{2} \times(0, \infty)$.i.e. $\lim _{n \rightarrow \infty} M\left(x_{n}, x, t\right)=\lim _{n \rightarrow \infty} M\left(y_{n}, y, t\right)=1$ and $\lim _{n \rightarrow \infty} M\left(x, y, t_{n}\right)=$ $M(x, y, t)$.

Lemma 1.12. ([3]). Let $(X, M, *)$ be a fuzzy metric space. Then $M$ is a continuous function on $X^{2} \times(0, \infty)$

Now we state the condition $(\mathrm{A}): \lim _{t \rightarrow \infty} M(x, y, t)=1$ for all $x, y \in X$.

We observed that in the proof of Theorem 1.6, the authors Murthy and Rashmi [8] inherently used the condition $(\mathrm{A})$.

Now we introduce the definition of jointly $2 k$-weakly compatible pairs as follows.

Definition 1.13. Let $X$ be a nonempty set, $k$ a positive integer and $S, T: X^{2 k} \rightarrow X$ and $f, g: X \rightarrow X$. The pairs $(f, S)$ and $(g, T)$ are said to be jointly $2 k$-weakly compatible if

$$
f(S(x, x, \ldots, x))=S(f x, f x, \ldots, f x)
$$

and

$$
g(T(x, x, \ldots, x))=T(g x, g x, \ldots, g x)
$$

whenever there exists $x \in X$ such that $f x=S(x, x, \ldots, x)$ and $g x=T(x, x, \ldots, x)$.

Now we give our main theorem.

\section{Main Result}

Throughout this section assume $\phi$ as in Definition 1.5

Theorem 2.1. Let $(X, M, *)$ be a fuzzy metric space with the condition $(A), k$ a positive integer and $S, T: X^{2 k} \longrightarrow X$ and $f, g: X \longrightarrow X$ be mappings satisfying:

(2.1.1) $S\left(X^{2 k}\right) \subseteq g(X), T\left(X^{2 k}\right) \subseteq f(X)$,

$$
\text { 1.2) } M\left(S\left(x_{1}, x_{2}, \ldots, x_{2 k}\right), T\left(y_{1}, y_{2}, \ldots, y_{2 k}\right), q t\right) \geq \phi\left(\begin{array}{c}
M\left(g x_{1}, f y_{1}, t\right), M\left(f x_{2}, g y_{2}, t\right), \\
M\left(g x_{3}, f y_{3}, t\right), M\left(f x_{4}, g y_{4}, t\right), \\
\vdots \\
M\left(g x_{2 k-1}, f y_{2 k-1}, t\right), M\left(f x_{2 k}, g y_{2 k}, t\right)
\end{array}\right)
$$


(2.1.3) $(f, S)$ and $(g, T)$ are jointly $2 k$-weakly compatible pairs.

(2.1.4) Suppose $z=f u=g u$ for some $u \in X$ whenever there exists a sequence $\left\{y_{2 k+n}\right\}_{n=1}^{\infty}$ in $X$ such that $\lim _{n \rightarrow \infty} y_{2 k+n}=z \in X$.

Then $z$ is the unique point in $X$ such that $z=f z=g z=S(z, z, . ., z, z)=T(z, z, \ldots, z, z)$.

Proof. Suppose $x_{1}, x_{2}, \ldots, x_{2 k}$ are arbitrary points in $X$. From (2.1.1),we define

$$
\begin{gathered}
y_{2 k+2 n-1}=S\left(x_{2 n-1}, x_{2 n}, \ldots, x_{2 k+2 n-2}\right)=g x_{2 k+2 n-1} \\
y_{2 k+2 n}=T\left(x_{2 n}, x_{2 n+1}, \ldots, x_{2 k+2 n-1}\right)=f x_{2 k+2 n}
\end{gathered}
$$

for $n=1,2, \ldots$

Let $\alpha_{2 n}=M\left(f x_{2 n}, g x_{2 n+1}, q t\right)$ and $\alpha_{2 n-1}=M\left(g x_{2 n-1}, f x_{2 n}, q t\right)$ for $n=1,2, \ldots$

Put $\theta=\frac{1}{q}$ and $\mu=\min \left\{\theta \frac{1+\sqrt{\alpha_{1}}}{1-\sqrt{\alpha_{1}}}, \theta^{2} \frac{1+\sqrt{\alpha_{2}}}{1-\sqrt{\alpha_{2}}}, \ldots, \theta^{2 k} \frac{1+\sqrt{\alpha_{2 k}}}{1-\sqrt{\alpha_{2 k}}}\right\}$. Then $\theta>1$.

By the selection of $\mu$, we have

$$
\alpha_{n} \geq\left(\frac{\mu-\theta^{n}}{\mu+\theta^{n}}\right)^{2} \text { for } n=1,2, \ldots, 2 k
$$

Consider

$$
\begin{aligned}
\alpha_{2 k+1} & =M\left(g x_{2 k+1}, f x_{2 k+2}, q t\right) \\
& =M\left(S\left(x_{1}, x_{2}, \ldots, x_{2 k-1}, x_{2 k}\right), T\left(x_{2}, x_{3}, \ldots, x_{2 k}, x_{2 k+1}\right), q t\right) \\
& \geq \phi\left(M\left(g x_{1}, f x_{2}, t\right), M\left(f x_{2}, g x_{3}, t\right), \ldots, M\left(f x_{2 k}, g x_{2 k+1}, t\right)\right) \\
& \geq \phi\left(\alpha_{1}, \alpha_{2}, \ldots, \alpha_{2 k-1}, \alpha_{2 k}\right), \text { since } M(x, y, .) \text { and } \phi \text { are increasing } \\
& \geq \phi\left(\left(\frac{\mu-\theta}{\mu+\theta}\right)^{2},\left(\frac{\mu-\theta^{2}}{\mu+\theta^{2}}\right)^{2}, \ldots,\left(\frac{\mu-\theta^{2 k}}{\mu+\theta^{2 k}}\right)^{2}\right) \text { from }(1) \\
& \geq \phi\left(\left(\frac{\mu-\theta^{2 k}}{\mu+\theta^{2 k}}\right)^{2},\left(\frac{\mu-\theta^{2 k}}{\mu+\theta^{2 k}}\right)^{2}, \ldots,\left(\frac{\mu-\theta^{2 k}}{\mu+\theta^{2 k}}\right)^{2}\right) \\
& \geq\left(\frac{\mu-\theta^{2 k}}{\mu+\theta^{2 k}}\right)^{2}, \text { since } \phi(t, t, \ldots, t) \geq t \\
& \geq\left(\frac{\mu-\theta^{2 k+1}}{\mu+\theta^{2 k+1}}\right)^{2} .
\end{aligned}
$$

Thus

$$
\alpha_{2 k+1} \geq\left(\frac{\mu-\theta^{2 k+1}}{\mu+\theta^{2 k+1}}\right)^{2}
$$

Also

$$
\begin{aligned}
\alpha_{2 k+2} & =M\left(f x_{2 k+2}, g x_{2 k+3}, q t\right) \\
& =M\left(S\left(x_{3}, x_{4}, x_{5}, x_{6}, \ldots, x_{2 k+1}, x_{2 k+2}\right), T\left(x_{2}, x_{3}, x_{4}, x_{5}, \ldots, x_{2 k}, x_{2 k+1}\right), q t\right) \\
& \geq \phi\left(M\left(g x_{3}, f x_{2}, t\right), M\left(f x_{4}, g x_{3}, t\right), \ldots, M\left(f x_{2 k+2}, g x_{2 k+1}, t\right)\right) \\
& \geq \phi\left(\alpha_{2}, \alpha_{3}, \alpha_{4}, \alpha_{5}, \ldots, \alpha_{2 k}, \alpha_{2 k+1}\right) \\
& \geq \phi\left(\left(\frac{\mu-\theta^{2}}{\mu+\theta^{2}}\right)^{2},\left(\frac{\mu-\theta^{3}}{\mu+\theta^{3}}\right)^{2}, \ldots,\left(\frac{\mu-\theta^{2 k}}{\mu+\theta^{2 k}}\right)^{2},\left(\frac{\mu-\theta^{2 k+1}}{\mu+\theta^{2 k+1}}\right)^{2}\right) \\
& \geq \phi\left(\left(\frac{\mu-\theta^{2 k+1}}{\mu+\theta^{2 k+1}}\right)^{2},\left(\frac{\mu-\theta^{2 k+1}}{\mu+\theta^{2 k+1}}\right)^{2}, \ldots,\left(\frac{\mu-\theta^{2 k+1}}{\mu+\theta^{2 k+1}}\right)^{2}\right) \\
& \geq\left(\frac{\mu-\theta^{2 k+1}}{\mu+\theta^{2 k+1}}\right)^{2} \geq\left(\frac{\mu-\theta^{2 k+2}}{\mu+\theta^{2 k+2}}\right)^{2} .
\end{aligned}
$$


Thus

$$
\alpha_{2 k+2} \geq\left(\frac{\mu-\theta^{2 k+2}}{\mu+\theta^{2 k+2}}\right)^{2}
$$

Continuing in this way,we have

$$
\alpha_{n} \geq\left(\frac{\mu-\theta^{n}}{\mu+\theta^{n}}\right)^{2}, n=1,2,3 \ldots
$$

Now consider

$M\left(y_{2 k+2 n-1}, y_{2 k+2 n}, t\right) \geq M\left(y_{2 k+2 n-1}, y_{2 k+2 n}, q t\right)$, since $q<1$ and $M(x, y,$.$) is increasing$

$$
\begin{aligned}
& =M\left(\begin{array}{l}
S\left(x_{2 n-1}, x_{2 n}, x_{2 n+1}, \ldots x_{2 k+2 n-3}, x_{2 k+2 n-2}\right), \\
T\left(x_{2 n}, x_{2 n+1}, x_{2 n+2}, \ldots x_{2 k+2 n-2}, x_{2 k+2 n-1}\right), q t
\end{array}\right)
\end{aligned}
$$

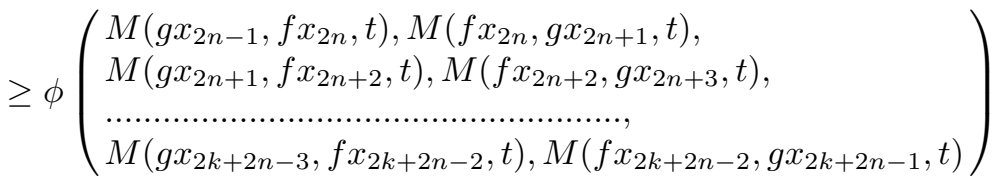

$\geq \phi\left(\alpha_{2 n-1}, \alpha_{2 n}, \alpha_{2 n+1}, \ldots, \alpha_{2 k+2 n-3}, \alpha_{2 k+2 n-2}\right)$, since $\phi$ and $M$ are increasing$$
\geq \phi\left(\left(\frac{\mu-\theta^{2 n-1}}{\mu+\theta^{2 n-1}}\right)^{2},\left(\frac{\mu-\theta^{2 n}}{\mu+\theta^{2 n}}\right)^{2}, \ldots,\left(\frac{\mu-\theta^{2 k+2 n-2}}{\mu+\theta^{2 k+2 n-2}}\right)^{2}\right) \text { from (4) }
$$$$
\geq \phi\left(\left(\frac{\mu-\theta^{2 k+2 n-2}}{\mu+\theta^{2 k+2 n-2}}\right)^{2},\left(\frac{\mu-\theta^{2 k+2 n-2}}{\mu+\theta^{2 k+2 n-2}}\right)^{2}, \ldots,\left(\frac{\mu-\theta^{2 k+2 n-2}}{\mu+\theta^{2 k+2 n-2}}\right)^{2}\right)
$$$$
\geq\left(\frac{\mu-\theta^{2 k+2 n-2}}{\mu+\theta^{2 k+2 n-2}}\right)^{2}
$$$$
\geq\left(\frac{\mu-\theta^{2 k+2 n-1}}{\mu+\theta^{2 k+2 n-1}}\right)^{2} \text {. }
$$

Thus

$$
M\left(y_{2 k+2 n-1}, y_{2 k+2 n}, t\right) \geq\left(\frac{\mu-\theta^{2 k+2 n-1}}{\mu+\theta^{2 k+2 n-1}}\right)^{2}
$$

Also

$M\left(y_{2 k+2 n}, y_{2 k+2 n+1}, t\right) \geq M\left(y_{2 k+2 n}, y_{2 k+2 n+1}, q t\right)$, since $q<1$ and $\phi$ is increasing

$$
\begin{aligned}
& =M\left(\begin{array}{l}
S\left(x_{2 n+1}, x_{2 n+2}, x_{2 n+3}, \ldots, x_{2 k+2 n-1}, x_{2 k+2 n}\right), \\
T\left(x_{2 n}, x_{2 n+1}, x_{2 n+2}, \ldots, x_{2 k+2 n-2}, x_{2 k+2 n-1}\right), q t
\end{array}\right),
\end{aligned}
$$

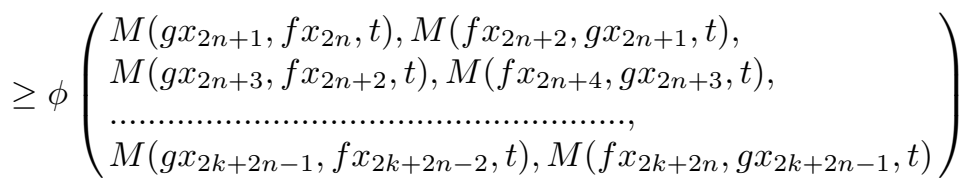

$$
\begin{aligned}
& \geq \phi\left(\alpha_{2 n}, \alpha_{2 n+1}, \ldots, \alpha_{2 k+2 n-2}, \alpha_{2 k+2 n-1}\right) \\
& \geq \phi\left(\left(\frac{\mu-\theta^{2 n}}{\mu+\theta^{2 n}}\right)^{2},\left(\frac{\mu-\theta^{2 n+1}}{\mu+\theta^{2 n+1}}\right)^{2}, \ldots,\left(\frac{\mu-\theta^{2 k+2 n-1}}{\mu+\theta^{2 k+2 n-1}}\right)^{2}\right) \text { from (4) } \\
& \geq \phi\left(\left(\frac{\mu-\theta^{2 k+2 n-1}}{\mu+\theta^{2 k+2 n-1}}\right)^{2},\left(\frac{\mu-\theta^{2 k+2 n-1}}{\mu+\theta^{2 k+2 n-1}}\right)^{2}, \ldots,\left(\frac{\mu-\theta^{2 k+2 n-1}}{\mu+\theta^{2 k+2 n-1}}\right)^{2}\right) \\
& \geq\left(\frac{\mu-\theta^{2 k+2 n-1}}{\mu+\theta^{2 k+2 n-1}}\right)^{2}, \text { since } \phi(t, t, t, \ldots, t) \geq t \\
& \geq\left(\frac{\mu-\theta^{2 k+2 n}}{\mu+\theta^{2 k+2 n}}\right)^{2}
\end{aligned}
$$


Thus

$$
M\left(y_{2 k+2 n}, y_{2 k+2 n+1}, t\right) \geq\left(\frac{\mu-\theta^{2 k+2 n}}{\mu+\theta^{2 k+2 n}}\right)^{2}
$$

Hence from (5) and (6)we have

$$
M\left(y_{2 k+n}, y_{2 k+n+1}, t\right) \geq\left(\frac{\mu-\theta^{2 k+n}}{\mu+\theta^{2 k+n}}\right)^{2} \text { for } n=1,2, \ldots
$$

Now for $n, p \in N$, we have

$$
\begin{aligned}
M\left(y_{2 k+n}, y_{2 k+n+p}, t\right) & \geq M\left(y_{2 k+n}, y_{2 k+n+1}, \frac{t}{p}\right) * M\left(y_{2 k+n+1}, y_{2 k+n+2}, \frac{t}{p}\right) * \ldots * M\left(y_{2 k+n+p-1}, y_{2 k+n+p}, \frac{t}{p}\right) \\
& \geq\left(\frac{\mu-\theta^{2 k+n}}{\mu+\theta^{2 k+n}}\right)^{2} *\left(\frac{\mu-\theta^{2 k+n+1}}{\mu+\theta^{2 k+n+1}}\right)^{2} * \ldots *\left(\frac{\mu-\theta^{2 k+n+p-1}}{\mu+\theta^{2 k+n+p-1}}\right)^{2}, \text { from }(7) \\
& \rightarrow 1 * 1 * 1 * \ldots * 1 \text { as } n \rightarrow \infty \\
& =1 .
\end{aligned}
$$

Hence $\left\{y_{2 k+n}\right\}$ is a Cauchy sequence in $X$.

Since $X$ is complete, there exists $z \in X$ such that $y_{2 k+n} \rightarrow z$ as $n \rightarrow \infty$.

From(2.1.4), there exists $u \in X$ such that

$$
z=f u=g u
$$

Now consider

$$
\begin{aligned}
& M\left(S(u, u, \ldots, u, u), y_{2 k+2 n}, q t\right)=M\left(S(u, u, \ldots, u, u), T\left(x_{2 n}, x_{2 n+1}, \ldots, x_{2 n+2 k-2}, x_{2 n+2 k-1}\right), q t\right)
\end{aligned}
$$

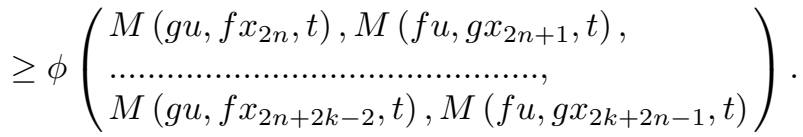

Letting $n \rightarrow \infty$ and using (8),we get

$$
M(S(u, u,, \ldots, u, u), f u, q t) \geq \phi(1,1, \ldots, 1,1) \geq 1
$$

which implies that

$$
S(u, u, \ldots, u, u)=f u
$$

Similarly we can prove that

$$
T(u, u, \ldots, u, u)=g u
$$

Since $(f, S)$ and $(g, T)$ are jointly $2 k$-weakly compatible pairs, we have

$$
f z=f(f u)=f(S(u, u, \ldots, u))=S(f u, f u, \ldots, f u)=S(z, z, \ldots, z)
$$

and also

$$
g z=T(z, z, \ldots, z, z)
$$

Now consider

$$
\begin{aligned}
& M(f z, z, q t)=M(S(z, z, \ldots, z, z), T(u, u, \ldots, u, u), q t), \text { from }(11),(8),(10)
\end{aligned}
$$

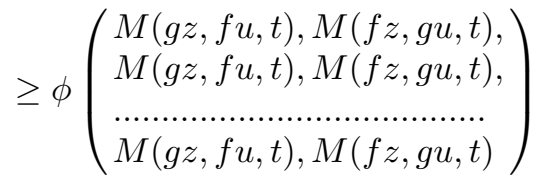

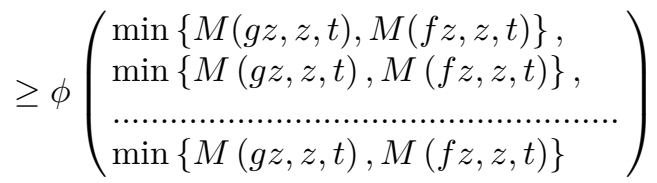

$$
\begin{aligned}
& \geq \min \{M(g z, z, t), M(f z, z, t)\} \text {. }
\end{aligned}
$$


Thus

$$
M(f z, z, q t) \geq \min \{M(g z, z, t), M(f z, z, t)\}
$$

Similarly,we can show that

$$
M(g z, z, q t) \geq \min \{M(z, f z, t), M(z, g z, t)\}
$$

Thus from (13)and (14), we have

$$
\min \{M(f z, z, q t), M(g z, z, q t)\} \geq \min \{M(z, f z, t), M(z, g z, t)\}
$$

which inturn yields from condition(A) that

$$
z=f z \text { and } z=g z
$$

From (11),(12)and (15),we have

$$
z=f z=g z=S(z, z, \ldots, z)=T(z, z, \ldots, z)
$$

Suppose there exists $z^{\prime} \in X$ such that

$$
z^{\prime}=f z^{\prime}=g z^{\prime}=S\left(z^{\prime}, z^{\prime}, \ldots, z^{\prime}, z^{\prime}\right)=T\left(z^{\prime}, z^{\prime}, \ldots, z^{\prime}, z^{\prime}\right)
$$

Then from (2.1.2) we have

$$
\begin{aligned}
& M\left(z, z^{\prime}, q t\right)=M\left(S(z, z, \ldots, z, z), T\left(z^{\prime}, z^{\prime}, \ldots, z^{\prime}, z^{\prime}\right), q t\right) \\
& \geq \phi\left(\begin{array}{l}
M\left(g z, f z^{\prime}, t\right), M\left(f z, g z^{\prime}, t\right), \\
M\left(g z, f z^{\prime}, t\right), M\left(f z, g z^{\prime}, t\right), \\
\cdots \ldots \ldots \ldots \ldots \ldots \ldots \ldots \ldots \ldots \ldots \ldots \\
M\left(g z, f z^{\prime}, t\right), M\left(f z, g z^{\prime}, t\right)
\end{array}\right) \\
& =\phi\left(M\left(z, z^{\prime}, t\right), M\left(z, z^{\prime}, t\right), \ldots, M\left(z, z^{\prime}, t\right)\right) \\
& \geq M\left(z, z^{\prime}, t\right)
\end{aligned}
$$

From the condition(A), we have $z^{\prime}=z$.

Thus $z$ is the unique point in $X$ satisfying (16).

Now we give an example to illustrate our main Theorem 2.1.

Example 2.2. Let $X=[0,1], a * b=a b, M(x, y, t)=e^{-\frac{|x-y|}{t}}$ and $k=1$. Define $\phi:[0,1]^{2} \rightarrow[0,1]$ as $\phi\left(x_{1}, x_{2}\right)=\min \left\{x_{1}, x_{2}\right\}$. Let $S, T: X^{2} \rightarrow X$ and $f, g: X \rightarrow X$ be defined as $S(x, y)=\frac{3 x^{2}+2 y}{72}, T(x, y)=$ $\frac{2 x+3 y^{2}}{72}, f x=\frac{x}{6}$ and $g x=\frac{x^{2}}{4}$. Now for $x_{1}, x_{2}, y_{1}, y_{2} \in X$, we have

$$
\begin{aligned}
\left|S\left(x_{1}, x_{2}\right)-T\left(y_{1}, y_{2}\right)\right| & =\left|\frac{3 x_{1}^{2}+2 x_{2}}{72}-\frac{2 y_{1}+3 y_{2}^{2}}{72}\right| \\
& =\frac{1}{72}\left|3 x_{1}^{2}-2 y_{1}+2 x_{2}-3 y_{2}^{2}\right| \\
& \leq \frac{1}{36} \max \left\{\left|3 x_{1}^{2}-2 y_{1}\right|,\left|2 x_{2}-3 y_{2}^{2}\right|\right\} .
\end{aligned}
$$

Now, we have

$$
\begin{aligned}
& M\left(S\left(x_{1}, x_{2}\right), T\left(y_{1}, y_{2}\right), \frac{1}{3} t\right)=e^{-\frac{\left|S\left(x_{1}, x_{2}\right)-T\left(y_{1}, y_{2}\right)\right|}{\frac{1}{3} t}} \\
& \geq e^{-\frac{1}{36} \frac{\max \left\{\left|3 x_{1}^{2}-2 y_{1}\right|,\left|2 x_{2}-3 y_{2}^{2}\right|\right\}}{\frac{t}{3}}} \\
& =e^{-\frac{\max \left\{\left|3 x_{1}^{2}-2 y_{1}\right|,\left|2 x_{2}-3 y_{2}^{2}\right|\right\}}{12 t}} \\
& =e^{-\frac{\max \left\{\left|\frac{x_{1}^{2}}{4}-\frac{y_{1}}{6}\right|,\left|\frac{x_{2}}{6}-\frac{y_{2}^{2}}{4}\right|\right\}}{t}} \\
& \geq \min \left\{e^{-\frac{\left|\frac{x_{1}^{2}}{4}-\frac{y_{1}}{6}\right|}{t}}, e^{-\frac{\left|\frac{x_{2}}{6}-\frac{y_{2}^{2}}{4}\right|}{t}}\right\} \\
& =\min \left\{M\left(g x_{1}, f y_{1}, t\right), M\left(f x_{2}, g y_{2}, t\right)\right\} \\
& =\phi\left(M\left(g x_{1}, f y_{1}, t\right), M\left(f x_{2}, g y_{2}, t\right)\right. \text {. }
\end{aligned}
$$

Thus (2.1.2) is satisfied with $q=\frac{1}{3}$.

One can easily verify the remaining conditions of Theorem 2.1. Clearly 0 is the unique point in $X$ satisfying (16). 
Corollary 2.3. Let $(X, M, *)$ be fuzzy metric space with the condition $(A)$ and $S, T: X^{2 k} \rightarrow X$ and $f: X \rightarrow X$ be mappings satisfying:

(2.3.1) $S\left(X^{2 k}\right) \subseteq f(X), T\left(X^{2 k}\right) \subseteq f(X)$,

$(2.3 .2) M\left(S\left(x_{1}, x_{2}, \ldots, x_{2 k}\right), T\left(y_{1}, y_{2}, \ldots, y_{2 k}\right), q t\right) \geq \phi\left(M\left(f x_{1}, f y_{1}, t\right), M\left(f x_{2}, f y_{2}, t\right), \ldots, M\left(f x_{2 k}, f y_{2 k}, t\right)\right)$ $\forall x_{1}, x_{2}, \ldots, x_{2 k}, y_{1}, y_{2}, \ldots, y_{2 k} \in X, \forall t>0$ and $0<q<1$,

(2.3.3) $f(X)$ is a complete subspace of $X$.

(2.3.4) Either $(f, S)$ or $(f, T)$ is a $2 k$-weakly compatible pair. Then there exists a unique $u \in X$ such that $u=f u=S(u, u, \ldots, u, u)=T(u, u, \ldots, u, u)$.

Corollary 2.4. Let $(X, M, *)$ be a complete fuzzy metric space with the condition $(A)$ and $S, T: X^{2 k} \rightarrow X$ be mappings satisfying:

(2.4.1) $M\left(S\left(x_{1}, x_{2}, \ldots, x_{2 k}\right), T\left(y_{1}, y_{2}, \ldots, y_{2 k}\right), q t\right) \geq \phi\left(M\left(x_{1}, y_{1}, t\right), M\left(x_{2}, y_{2}, t\right), \ldots, M\left(x_{2 k}, y_{2 k}, t\right)\right)$ $\forall x_{1}, x_{2}, \ldots, x_{2 k}, y_{1}, y_{2}, \ldots, y_{2 k} \in X, \forall t>0$ and $0<q<1$.

Then there exists a unique $u \in X$ such that $u=S(u, u, \ldots, u)=T(u, u, \ldots, u)$.

\section{References}

1. A.George and P.Veeramani,On some results in fuzzy metric spaces, Fuzzy sets and Systems, 64(3), (1994), $395-399$.

2. B.Schweizer and A.Sklar, Statistical metric spaces, Pacific J.Math., 10(1),(1960),313-334.

3. J.Rodríguez López and S.Romaguera, The Hausdorff fuzzy metric on compact sets, Fuzzy sets and systems, 147(2), (2004), $273-283$.

4. K.P.R.Rao, G.N.V.Kishore and Md.Mustaq Ali, Generalization of Banach contraction principle of Presic type for three maps, Math.Sci. 3(3), 2009, 273 - 280.

5. K.P.R.Rao, Md.Mustaq Ali and B.Fisher, Some Presic type generalization of Banach contraction principle, Math.Moravica 15(2011), 41 - 47.

6. Lj.B.Ciric and S.B.Presic, On Presic type generalization of Banach contraction mapping principle, Acta.Math.Univ.Comenianae, LXXVI (2), (2007), 143-147.

7. M.Grabiec, Fixed points in fuzzy metric space, Fuzzy sets and Systems, 27(3), (1988), 385 - 389.

8. P.P.Murthy and Rashmi,A common fixed point theorem of Presic type for three maps in fuzzy metric space,Annual Review of Chaos Theory,Bifurcations and Dynamical Systems,4(2013),30-36.

9. S.B.Presic, Sur une classe $d^{1}$ inequations aux differences finite et sur la convergence de certaines suites, Pub de $l^{1}$ inst. Math. Belgrade, 5(19), (1965), 75-78. 